\title{
Microstructures of Concrete: Evolution with Hardness
}

\author{
Federico Sebastián Cuenca ${ }^{1}$ and María del Rosario Torres Deluigi ${ }^{1,2, *}$ \\ 1. Instituto de Química San Luis, CCT-SL, CONICET - Universidad Nacional de San Luis (UNSL), San \\ Luis, Argentina \\ 2. Departamento de Física, Facultad de Ciencias Físico, Matemáticas y Naturales, UNSL, San Luis, \\ Argentina \\ * Corresponding author: charo@unsl.edu.ar
}

The microstructures of concrete evolve over time, therefore, the behavior of the concrete put in construction, particularly its hardness, depends of its age. This is due to chemical reactions of Portland cement hydration, mainly during the setting period, although they continue later during hardening, given that while there is moisture the concrete will continue to change its internal structure although at a lower speed. In turn, it also depends on other factors such as curing conditions, and exposure to variety of environmental causes, chemical, physical and biological.

The understanding of the mechanism of the hydration processes and chemical reactions of cement hydrolysis will contribute to the explanation of the anomalies in the bonding and hardening process. The strength of the hardened cement paste is a function of the mineral and chemical composition, as well as the structure of the crystalline lattice [1,2]. Therefore, studies of crystallization of the mineral phases of the different hydration products and observations of the reconstruction of the maturation microstructure of the cement paste [3-4] are needed. The method proposed in this work to study this type of phenomena consists in the use of Scanning Electron Microscopy (SEM) combined with the microanalysis of the chemical composition by means of Energy Dispersive Spectrometer (EDS). So, the results of mineralogical microstructure study of concretes with different hardness are presented.

In this work three concretes of different ages (2, 8 and 33 days) were analyzed, they cover the range of $30 \%$ to $100 \%$ hardness, so that the changes found in the crystalline structures are representative of the evolution experienced by the concrete throughout its hardening process. The samples were collected after performing the compression test of concrete samples, performed according to the corresponding international norms. Then, a strong dependence between the concrete hardness and the development of crystalline microstructures was found. For example, in the hardest concrete, regions with massive formation of massive Ettringite were observed covering the surface, whereas other regions presented a well-compacted amorphous background of Tobermorite gel. From the morphological point of view, these results provide evidence of a mature concrete. In addition, the microanalysis performed with EDS allowed identifying the different mineral phases, associating the morphology observed by SEM with the chemical composition.

In general, it was found that the hydration process occurs differently in each mineral, which was evidenced in the crystalline changes experimented by them during the transformation of the anhydrous phase to the hydrated phase. A complete chemical compositional analysis of the Portland cement hydration product was obtained, verifying the presence and evolution of different crystalline phases, such as Tobermorite gel, Portlandite and Ettringite, during the cure time, as well as the presence of calcium monosulfoaluminate and hydrated tricalcium aluminate in older concretes (see Figure 1 and Figure 2). 
It should be noted that the concretes under study in this work have not been part of building in which they would be exposed to action of different factors (environmental conditions, acid attack, corrosion in reinforced concrete, etc.) that would modify their internal microscopic structure and therefore their hardness .The knowing and characterizing of these concretes not exposed to building is very important because they could be use to compare with those that certainly have undergone changes in their hardness after to their exposed to the construction, and thereby interpret why occurred such changes [5].

\section{References}

[1] M Arandigoyen and J I Alvarez, Cement and Concrete Research 37 (2007), p. 767.

[2] W Franus, R Panek and M Wdowin, 2nd International Multidisciplinary Microscopy and Microanalysis Congress, Springer Proceedings in Physics 164 (2015), p. 105.

[3] J Calleja. Cemento y Hormigón 72 (2001), p. 526.

[4] M A Giraldo and J I Tobón, Dyna 73 (2006), p. 69.

[5] The authors thank the services provided by the Laboratorio de Microscopía Electrónica y Microanálisis (LABMEM) of Universidad Nacional de San Luis in which the analyzes of this study were performed.
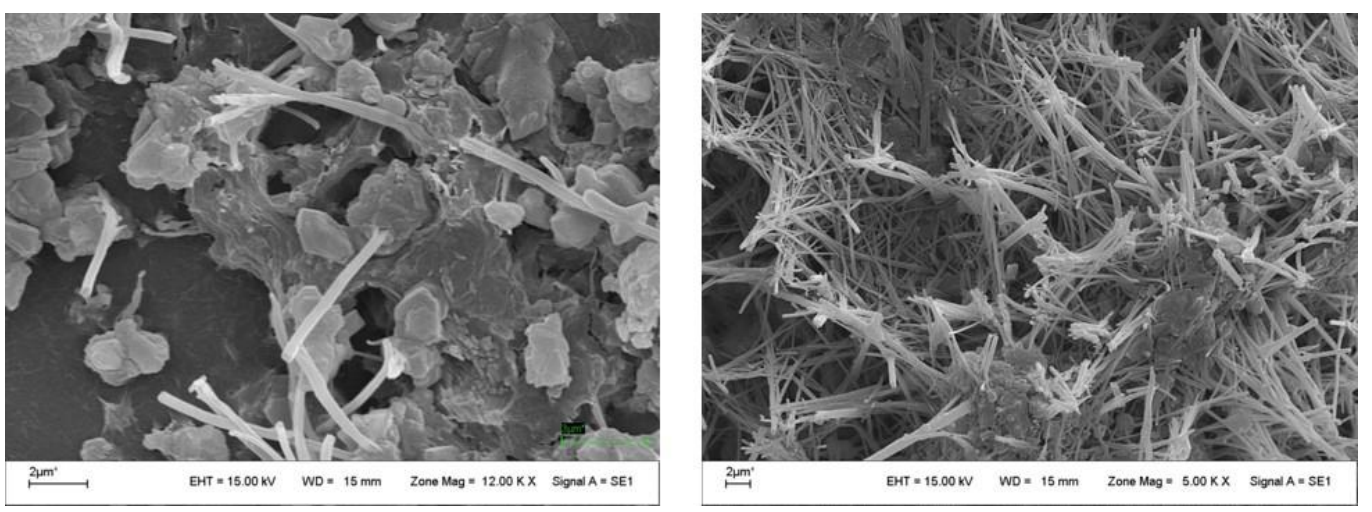

Figure 1. Left: cubic crystals of hydrated tricalcium aluminate. Right: crystals of Ettringite.

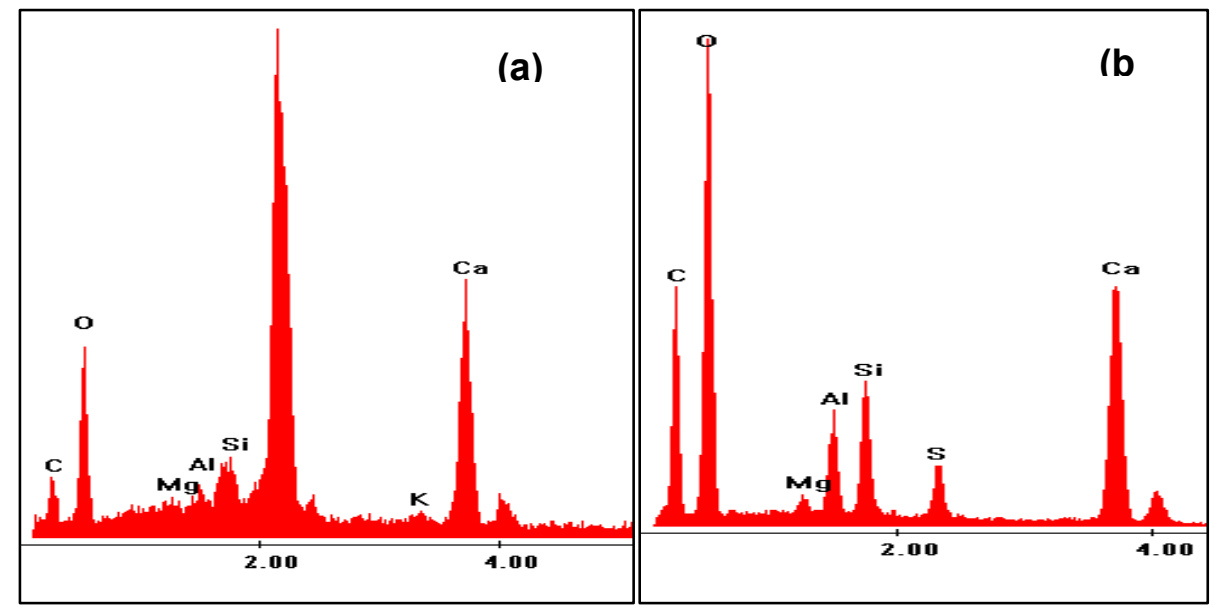

Figure 2. (a): EDS Spectrum of hydrated tricalcium aluminate. (b): EDS Spectrum of Ettringite. 